\title{
Synthesis of Pyrrolo[3, 4-d]Pyrimidine Thiono Derivatives via Aza Wittig Reaction
}

\author{
Hussain Ali Soleiman ${ }^{1}$ \\ ${ }^{1}$ Chemistry Department, Aswan Faculty of Science, Aswan University, Egypt \\ Correspondence: Hussain Ali Soleiman, Chemistry Department, Aswan Faculty of Science, Aswan University, \\ Egypt. Tel: 2-97-344-7480. Fax: 2-97-344-7450. E-mail: hsoleiman2001@ yahoo.com
}

Received: September 9, 2014 Accepted: October 16, 2014 Online Published: October 28, 2014

doi:10.5539/ijc.v6n4p55

URL: http://dx.doi.org/10.5539/ijc.v6n4p55

\begin{abstract}
New pyrolo [3,4-d] pyrimidine derivatives were obtained from the aza-Wittig reactions of iminophosphorane with phenylisocyanates via of amino compounds with triphenylphosphine, hexachloroethane and triethyl-amine produced iminophosphorane.
\end{abstract}

Keywords: amino compound,isocyanate, pyrrole, pyrimidine, aza-Wittig reaction, synthesis

\section{Introduction}

The aza-Wittig reactions of iminophosphoranes have received increased attention in view of their utility in the synthesis of nitrogen heterocyclic compounds (Palacios et al., 2006, 2007; Lopez et al., 2007; Lertpibulpanya et al., 2006; Marsden et al., 2008; Haraguchi et al., 2004; Cushman et al., 2004; Depecker et al., 2004; Wnuk et al., 2004). The reaction is useful in the synthesis of acyclic imines and heterocumulenes and in the intramolecular formation of carbon-nitrogen double bonds in heterocyclic synthesis. Stability, basicity, and nucleophilicity of iminophosphoranes are mainly determined by the substitutes at the nitrogen atom. Carbonyl groups of aldehydes, ketones, acid halides, and heterocumulenes are generally reactive (Daboun et al., 1983). Recently we have been interested in the synthesis of new pyrrolo [3,4-d] pyrimidine derivatives via aza-Wittig reaction by pyrimidines nuclei with phenylisocyanate (He et al., 2013, 2014). Pyrimidine derivatives play an important role in several biological and pharmacological active substances such as antibacterial and antitumor agents as well as agrochemical and veterinary products (Wright et al., 1977; Taylor et al., 1992; Traxler et al., 1997; Vicentini et al., 1989; Molina et al., 1994). The nucleophilicity at the nitrogen is a factor of essential mechanistic importance in the use of these iminophosphoranes as aza-Wittig reagents. Iminophosphoranes are important reagents in synthetic organic chemistry, especially in the synthesis of naturally occurring products, compounds with biological and pharmacological activity (Ramazani et al., 2008). Organophosphorus compounds have been extensively employed in organic synthesis as useful reagents, as well as legends, in a number of transition metal catalysts (Kaska, 1983; Ramazani et al., 2006; Stolzenberg et al., 2005).

\section{Experimental}

\subsection{Synthesis of 6-Amino-4-Hydroxy-2-Thiouracil Sodium Salt Derivatives (1a-f)}

To a solution of $4.6 \mathrm{mg}(0.2 \mathrm{Mol})$ of metallic sodium in $75 \mathrm{ml}$ of absolute ethanol was added $7.6 \mathrm{gm}(0.1 \mathrm{Mol})$ of powdered thiourea and arylidenocyano-ethyl-acetate (cinnamonitrile) ylidenocyanoethylacetate. The reaction mixture was heated under reflux $(0.1 \mathrm{~mol})$ for two hours, then filtered while hot. The sodium salt of 6-amino-4-hydroxy-2-thiouracil thus obtained was washed alcohol and dried.

\subsection{Synthesis of 6-Amino-4-Hydroxy-2-Thiouracil Derivatives (2a-f)}

To a cold solution sodium salt of 6-amino-4-hydroxy-2-thiouracil derivatives (1a-f) in ice-water was added dilute $\mathrm{HCl}$ until the mixture was neutralized, whereby crystalline products separated out, and crystallized from the appropriate solvent (ethanol) to give 6-amino-4-hydroxy-2-thiouracil derivatives (2a-f). The results are listed in Table 1.

\subsection{Synthesis of 4-Hydroxy-6-Aminotriphenylphosphorane-2-Thiouracil (3a-f)}

To a mixture of 6-amino-4-hydroxy-2-thiouracil derivatives 2a-f (1.56, 1.71, 2.22, 2.332.62, 8 mmol), triphenylphosphine $(3.14 \mathrm{~g}, 12 \mathrm{mmol})$ and hexachloroethan $(2.84 \mathrm{~g}, 12 \mathrm{mmol})$ in dry acetonitrile $(40 \mathrm{ml})$ was 
Table 1. Characterization of compounds (1-5) a-f

\begin{tabular}{|c|c|c|c|c|c|c|c|c|c|c|}
\hline \multirow{2}{*}{$\begin{array}{l}\text { Comp. } \\
\text { No. }\end{array}$} & \multirow{2}{*}{$\begin{array}{l}\text { M.P. } \\
{ }^{\circ} \mathrm{C}\end{array}$} & \multirow{2}{*}{ Colour } & \multirow{2}{*}{$\begin{array}{c}\text { Yield } \\
\%\end{array}$} & \multirow{2}{*}{ Cryst. } & \multirow{2}{*}{$\begin{array}{c}\text { M.F } \\
\text { (M. Wt.) }\end{array}$} & \multicolumn{4}{|c|}{ Analysis \% calced (found) } & \multirow{2}{*}{ M.S } \\
\hline & & & & & & $\mathrm{C}$ & $\mathrm{H}$ & $\mathrm{N}$ & $\mathrm{S}$ & \\
\hline $1 \mathrm{a}$ & $>300$ & Yellow & 40 & Ethanol & $\begin{array}{c}\mathrm{C}_{5} \mathrm{H}_{6} \mathrm{~N}_{3} \mathrm{OSNa} \\
(179.17)\end{array}$ & $\begin{array}{l}33.52 \\
(33.50)\end{array}$ & $\begin{array}{c}3.38 \\
(3.38)\end{array}$ & $\begin{array}{c}23.45 \\
(23.48)\end{array}$ & $\begin{array}{c}17.89 \\
(17.85)\end{array}$ & 179 \\
\hline $1 \mathrm{~b}$ & $>300$ & $\begin{array}{c}\text { Pale } \\
\text { yellow }\end{array}$ & 50 & Ethanol & $\begin{array}{c}\mathrm{C}_{6} \mathrm{H}_{9} \mathrm{~N}_{3} \mathrm{OSNa} \\
(194.20)\end{array}$ & $\begin{array}{c}37.11 \\
(37.12)\end{array}$ & $\begin{array}{c}4.67 \\
(4.66)\end{array}$ & $\begin{array}{c}21.64 \\
(21.70)\end{array}$ & $\begin{array}{c}16.51 \\
(16.56)\end{array}$ & 194 \\
\hline $1 \mathrm{c}$ & $>300$ & Brown & 80 & Ethanol & $\begin{array}{c}\mathrm{C}_{9} \mathrm{H}_{8} \mathrm{~N}_{3} \mathrm{O}_{2} \mathrm{SNa} \\
(245.23)\end{array}$ & $\begin{array}{c}44.08 \\
(44.10)\end{array}$ & $\begin{array}{c}3.29 \\
(3.30)\end{array}$ & $\begin{array}{c}17.13 \\
(17.15)\end{array}$ & $\begin{array}{c}13.07 \\
(13.12)\end{array}$ & 245 \\
\hline $1 \mathrm{~d}$ & $>300$ & White & 70 & Ethanol & $\begin{array}{c}\mathrm{C}_{11} \mathrm{H}_{11} \mathrm{~N}_{3} \mathrm{OSNa} \\
(256.27)\end{array}$ & $\begin{array}{c}51.55 \\
(51.56)\end{array}$ & $\begin{array}{c}4.33 \\
(4.32)\end{array}$ & $\begin{array}{c}16.40 \\
(16.44)\end{array}$ & $\begin{array}{c}12.51 \\
(12.50)\end{array}$ & 256 \\
\hline $1 \mathrm{f}$ & $>300$ & Yellow & 85 & Ethanol & $\begin{array}{c}\mathrm{C}_{12} \mathrm{H}_{12} \mathrm{~N}_{3} \mathrm{O}_{2} \mathrm{SNa} \\
(285.29)\end{array}$ & $\begin{array}{c}50.52 \\
(50.48)\end{array}$ & $\begin{array}{l}4.24 \\
(4.22)\end{array}$ & $\begin{array}{c}14.73 \\
(14.75)\end{array}$ & $\begin{array}{c}11.24 \\
(11.30)\end{array}$ & 285 \\
\hline $2 \mathrm{a}$ & $218-220$ & Yellow & 40 & Ethanol & $\begin{array}{c}\mathrm{C}_{5} \mathrm{H}_{6} \mathrm{~N}_{3} \mathrm{OS} \\
(156.18)\end{array}$ & $\begin{array}{c}38.45 \\
(38.45)\end{array}$ & $\begin{array}{c}3.87 \\
(3.88)\end{array}$ & $\begin{array}{c}26.90 \\
(26.86)\end{array}$ & $\begin{array}{c}20.53 \\
(20.48)\end{array}$ & 156 \\
\hline $2 b$ & $200-202$ & $\begin{array}{c}\text { Pale } \\
\text { yellow }\end{array}$ & 50 & Ethanol & $\begin{array}{c}\mathrm{C}_{6} \mathrm{H}_{9} \mathrm{~N}_{3} \mathrm{OS} \\
(171.21)\end{array}$ & $\begin{array}{c}42.09 \\
(42.12)\end{array}$ & $\begin{array}{c}5.30 \\
(5.30)\end{array}$ & $\begin{array}{c}24.54 \\
(24.50)\end{array}$ & $\begin{array}{c}18.72 \\
(18.75)\end{array}$ & 171 \\
\hline $2 \mathrm{c}$ & $244-246$ & White & 80 & Ethanol & $\begin{array}{c}\mathrm{C}_{9} \mathrm{H}_{8} \mathrm{~N}_{3} \mathrm{O}_{2} \mathrm{~S} \\
(222.24)\end{array}$ & $\begin{array}{c}48.64 \\
(48.66)\end{array}$ & $\begin{array}{c}3.63 \\
(3.62)\end{array}$ & $\begin{array}{c}18.91 \\
(18.94)\end{array}$ & $\begin{array}{c}14.43 \\
(14.48)\end{array}$ & 222 \\
\hline $2 d$ & 187-189 & Yellow & 70 & Ethanol & $\begin{array}{c}\mathrm{C}_{11} \mathrm{H}_{11} \mathrm{~N}_{3} \mathrm{OS} \\
(233.28)\end{array}$ & $\begin{array}{c}56.63 \\
(56.60)\end{array}$ & $\begin{array}{c}4.75 \\
(4.76)\end{array}$ & $\begin{array}{c}18.01 \\
(18.05)\end{array}$ & $\begin{array}{c}13.74 \\
(13.70)\end{array}$ & 233 \\
\hline $2 f$ & $>300$ & Yellow & 90 & Ethanol & $\begin{array}{c}\mathrm{C}_{12} \mathrm{H}_{12} \mathrm{~N}_{3} \mathrm{O}_{2} \mathrm{~S} \\
(262.30)\end{array}$ & $\begin{array}{c}54.95 \\
(54.90)\end{array}$ & $\begin{array}{l}4.61 \\
(4.60)\end{array}$ & $\begin{array}{c}16.02 \\
(16.06)\end{array}$ & $\begin{array}{c}12.22 \\
(12.28)\end{array}$ & 262 \\
\hline $3 a$ & $123-125$ & $\begin{array}{c}\text { Pale } \\
\text { yellow }\end{array}$ & 20 & Ethanol & $\begin{array}{c}\mathrm{C}_{23} \mathrm{H}_{20} \mathrm{~N}_{3} \mathrm{OSP} \\
(417.46)\end{array}$ & $\begin{array}{c}66.17 \\
(66.20)\end{array}$ & $\begin{array}{c}4.83 \\
(4.80)\end{array}$ & $\begin{array}{c}10.07 \\
(10.12)\end{array}$ & $\begin{array}{c}7.68 \\
(7.64)\end{array}$ & 417 \\
\hline $3 b$ & $144-146$ & White & 40 & Ethanol & $\begin{array}{c}\mathrm{C}_{23} \mathrm{H}_{22} \mathrm{~N}_{3} \mathrm{OSP} \\
(419.48)\end{array}$ & $\begin{array}{c}65.86 \\
(65.84)\end{array}$ & $\begin{array}{c}5.29 \\
(5.30)\end{array}$ & $\begin{array}{c}10.02 \\
(10.06)\end{array}$ & $\begin{array}{c}7.64 \\
(7.70)\end{array}$ & 419 \\
\hline $3 c$ & $133-135$ & $\begin{array}{c}\text { Brownish } \\
\text { yellow }\end{array}$ & 50 & Ethanol & $\begin{array}{c}\mathrm{C}_{27} \mathrm{H}_{22} \mathrm{~N}_{3} \mathrm{OSP} \\
(467.52)\end{array}$ & $\begin{array}{c}69.36 \\
(69.35)\end{array}$ & $\begin{array}{c}4.74 \\
(4.74)\end{array}$ & $\begin{array}{c}8.99 \\
(9.00)\end{array}$ & $\begin{array}{c}6.86 \\
(6.90)\end{array}$ & 467 \\
\hline $3 d$ & $168-170$ & White & 40 & Ethanol & $\begin{array}{c}\mathrm{C}_{29} \mathrm{H}_{23} \mathrm{~N}_{3} \mathrm{OSP} \\
(492.55)\end{array}$ & $\begin{array}{c}70.42 \\
(70.40)\end{array}$ & $\begin{array}{l}4.71 \\
(4.70)\end{array}$ & $\begin{array}{c}8.53 \\
(8.50)\end{array}$ & $\begin{array}{c}6.51 \\
(6.46)\end{array}$ & 492 \\
\hline $3 f$ & $283-285$ & White & 60 & Ethanol & $\begin{array}{c}\mathrm{C}_{24} \mathrm{H}_{22} \mathrm{~N}_{3} \mathrm{OSP} \\
(431.49)\end{array}$ & $\begin{array}{c}66.81 \\
(66.78)\end{array}$ & $\begin{array}{c}5.14 \\
(5.10)\end{array}$ & $\begin{array}{r}9.74 \\
(9.78)\end{array}$ & $\begin{array}{c}7.43 \\
(7.40)\end{array}$ & 431 \\
\hline $4 \mathrm{a}$ & $114-116$ & $\begin{array}{c}\text { Pale } \\
\text { yellow }\end{array}$ & 60 & Ethanol & $\begin{array}{c}\mathrm{C}_{12} \mathrm{H}_{10} \mathrm{~N}_{4} \mathrm{OS} \\
(258.29)\end{array}$ & $\begin{array}{c}55.80 \\
(55.80)\end{array}$ & $\begin{array}{c}3.90 \\
(3.90)\end{array}$ & $\begin{array}{c}21.69 \\
(21.70)\end{array}$ & $\begin{array}{c}12.41 \\
(12.40)\end{array}$ & 258 \\
\hline $4 \mathrm{~b}$ & $162-164$ & $\begin{array}{c}\text { Pale } \\
\text { yellow }\end{array}$ & 20 & Ethanol & $\begin{array}{c}\mathrm{C}_{13} \mathrm{H}_{12} \mathrm{~N}_{4} \mathrm{OS} \\
(272.32)\end{array}$ & $\begin{array}{c}57.34 \\
(57.34)\end{array}$ & $\begin{array}{c}4.44 \\
(4.44)\end{array}$ & $\begin{array}{c}20.57 \\
(20.50)\end{array}$ & $\begin{array}{c}11.77 \\
(11.70)\end{array}$ & 272 \\
\hline $4 \mathrm{c}$ & $154-156$ & $\begin{array}{c}\text { Pale } \\
\text { yellow }\end{array}$ & 45 & Ethanol & $\begin{array}{c}\mathrm{C}_{16} \mathrm{H}_{11} \mathrm{~N}_{4} \mathrm{O}_{2} \mathrm{~S} \\
(323.34)\end{array}$ & $\begin{array}{c}59.43 \\
(59.45)\end{array}$ & $\begin{array}{c}3.43 \\
(3.40)\end{array}$ & $\begin{array}{c}17.33 \\
(17.35)\end{array}$ & $\begin{array}{l}9.91 \\
(9.90)\end{array}$ & 324 \\
\hline $4 d$ & $149-151$ & White & 20 & Ethanol & $\begin{array}{c}\mathrm{C}_{18} \mathrm{H}_{14} \mathrm{~N}_{4} \mathrm{OS} \\
(334.39)\end{array}$ & $\begin{array}{c}64.65 \\
(64.68)\end{array}$ & $\begin{array}{l}4.22 \\
(4.20)\end{array}$ & $\begin{array}{c}16.75 \\
(16.70)\end{array}$ & $\begin{array}{l}9.59 \\
(9.65)\end{array}$ & 334 \\
\hline $4 \mathrm{f}$ & $259-261$ & $\begin{array}{c}\text { Pale } \\
\text { yellow }\end{array}$ & 35 & Ethanol & $\begin{array}{c}\mathrm{C}_{19} \mathrm{H}_{16} \mathrm{~N}_{4} \mathrm{O}_{2} \mathrm{~S} \\
(364.42)\end{array}$ & $\begin{array}{c}62.62 \\
(62.60)\end{array}$ & $\begin{array}{c}4.43 \\
(4.44)\end{array}$ & $\begin{array}{c}15.37 \\
(15.40)\end{array}$ & $\begin{array}{c}8.80 \\
(8.86)\end{array}$ & 364 \\
\hline
\end{tabular}


added dropwise of tiethylamine $(2.42 \mathrm{~g}, 24 \mathrm{mmol})$ at room temperature. After for $6 \mathrm{~h}$, the solvent was removed under reduced pressure and the residue was recrystallized from EtOH to give iminophosphorane (3a-f).

\subsection{Synthesis of Pyrrolo [3,4-D] Pyrimidine Derivatives (5a-f)}

With a mixture of iminophosphorane derivatives (3a-f) $(4.17,4.19,4.92,4.92,4.31$ respectively). (10 mmol) in dry methylene dichloride $(25 \mathrm{ml})$ was added phenyl isocyanate $(10 \mathrm{mmol})$ under nitrogen at room temperature. After the reaction mixture was standing $8-10$ hours at $0-5^{\circ} \mathrm{C}$, the solvent was removed off under reduce pressure and ether (petroleum ether $(1: 2,20 \mathrm{ml})$ was added to precipitate triphenylphosphine oxide. Filtered, the solvent was removed to give carbodiimide derivatives, which were directly cyclized to pyrrolo [3,4-d] pyrimide derivatives (4a-f).

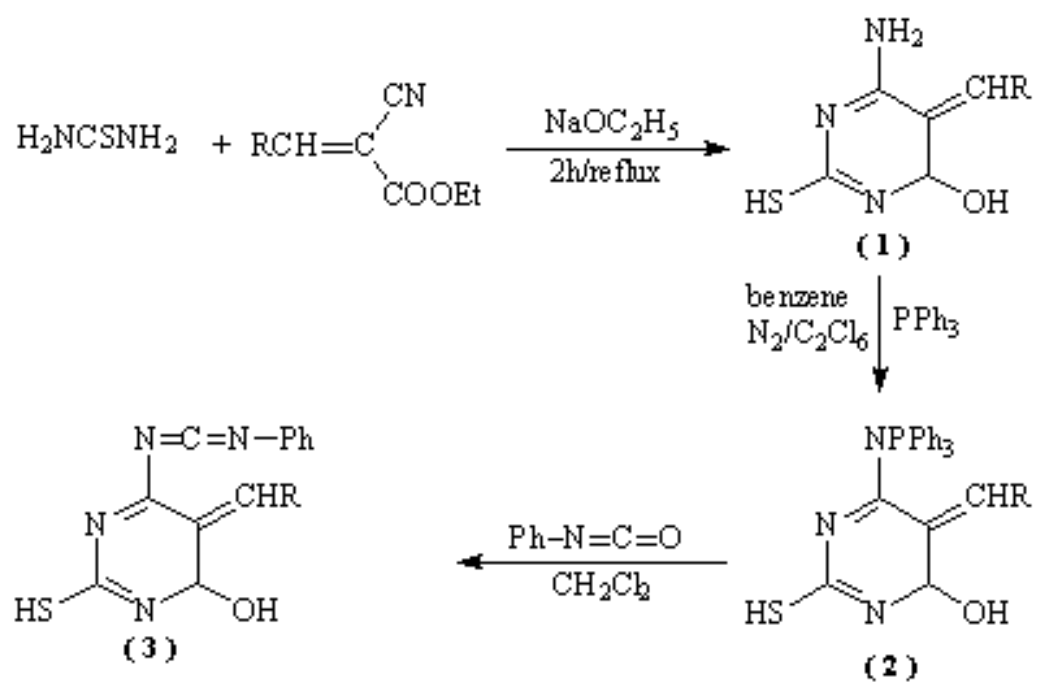<smiles>NN</smiles>

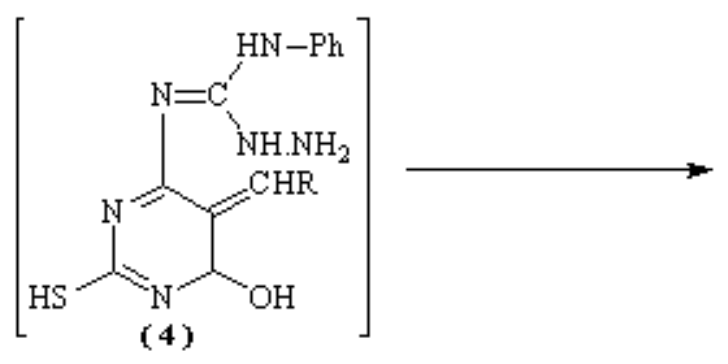<smiles>[R]C1=C2C(=NC(S)=[N+]([As])C2O)N=C(Nc2ccccc2)N1</smiles>

Where $(1,2,3,4)$ a-f: $a, \mathrm{R}=\mathrm{H} ; \mathrm{b}, \mathrm{R}=\mathrm{CH}_{3^{-}} ; \mathrm{c}, \mathrm{R}=\mathrm{C}_{6} \mathrm{H}_{5^{-}} ; \mathrm{d}, \mathrm{R}=\mathrm{p}-\mathrm{CH}_{3} \mathrm{O}-\mathrm{C}_{6} \mathrm{H}_{4} ; \mathrm{f}, \mathrm{R}=\mathrm{C}_{4} \mathrm{H}_{3} \mathrm{O}$.

\section{Results and Discussion}

The 6-amino-5-alkyl or/aryl-4-hydroxy-2-mercapto pyrimidine derivatives 1a-f was obtained by cyclization of alkylidine or/arylidine with thiourea under basic condition. The structure of compounds $1 \mathrm{a}-\mathrm{f}$ were confirmed by elemental analysis (c.f. Table 1), and IR spectrum $(\gamma \mathrm{KBr}$ ) showed general absorption bands at $3500 \mathrm{~cm}-1(\mathrm{OH})$, and at 3370-3350 cm-1 (NH2). The 1H NMR spectrum (DMSO)[10] of compounds 1a-f showed signals. 1a: at $9.0 \mathrm{ppm}(\mathrm{s}, 1 \mathrm{H}, \mathrm{OH}), 8.7 \mathrm{ppm}(\mathrm{s}, 1 \mathrm{H}, \mathrm{SH})$, at $8.5 \mathrm{ppm}(\mathrm{s}, 1 \mathrm{H}, \mathrm{Hpyrimidine}), 6 \mathrm{ppm}(\mathrm{s}, 2 \mathrm{H}, \mathrm{CH} 2)$, at $5.2 \mathrm{ppm}(\mathrm{br}, 2 \mathrm{H}$, $\mathrm{NH} 2)$. $1 \mathrm{~b}$ : at $9.0 \mathrm{ppm}(\mathrm{s}, 1 \mathrm{H}, \mathrm{OH})$, at $8.7 \mathrm{ppm}(\mathrm{s}, 1 \mathrm{H}, \mathrm{SH})$, at $8.5 \mathrm{ppm}(\mathrm{s}, 1 \mathrm{H}, \mathrm{Hpyrimidine})$, at $6 \mathrm{ppm}(\mathrm{s}, 1 \mathrm{H}, \mathrm{CH})$, at $5.2 \mathrm{ppm}(\mathrm{br}, 2 \mathrm{H}, \mathrm{NH} 2)$, at $2.3 \mathrm{ppm}(\mathrm{s}, 3 \mathrm{H}, \mathrm{CH} 3)$. 1c: at $9.0 \mathrm{ppm}(\mathrm{s}, 1 \mathrm{H}, \mathrm{OH})$, at $8.7 \mathrm{ppm}(\mathrm{s}, 1 \mathrm{H}, \mathrm{SH})$, at 8.5 $\operatorname{ppm}(\mathrm{s}, 1 \mathrm{H}, \mathrm{Hpyrimidine})$, at $6 \mathrm{ppm}(\mathrm{s}, 2 \mathrm{H}, \mathrm{CH} 2)$, at $5.2 \mathrm{ppm}(\mathrm{br}, 2 \mathrm{H}, \mathrm{NH} 2), 1 \mathrm{~b}:$ at $9.0 \mathrm{ppm}(\mathrm{s}, 1 \mathrm{H}, \mathrm{OH})$, at 8.7 ppm(s, $1 \mathrm{H}, \mathrm{SH})$, at $8.5 \mathrm{ppm}(\mathrm{s}, 1 \mathrm{H}, \mathrm{Hpyrimidine})$, at $8.3-7 \mathrm{ppm}(\mathrm{m}, 5 \mathrm{H}, \mathrm{Ar}-\mathrm{H}+)$, at $6 \mathrm{ppm}(\mathrm{s}, 1 \mathrm{H}, \mathrm{CH})$, at 5.2 $\mathrm{ppm}(\mathrm{br}, 2 \mathrm{H}, \mathrm{NH} 2)$. Thinopyrimidine derivatives 1a-f were converted to iminophosphorane $2 \mathrm{a}-\mathrm{f}$ via reaction with triphenylphosphine, hexachloroethane and triethylamine (Scheme). The structure of compounds $2 \mathrm{a}-\mathrm{f}$ were 
confirmed by elemental analysis (c.f. Table 1), and IR spectrum (KBr), general absorption band at $3500 \mathrm{~cm}^{-1}$ $(\mathrm{OH})$. The 1H NMR spectrum (DMSO)[10] of compounds 2a-f showed signals, 2a: at $9.0 \mathrm{ppm}(\mathrm{s}, 1 \mathrm{H}, \mathrm{OH})$, at 8.7 $\mathrm{ppm}(\mathrm{s}, 1 \mathrm{H}, \mathrm{SH})$, at $8.5 \mathrm{ppm}(\mathrm{s}, 1 \mathrm{H}, \mathrm{Hpyrimidine}), 6 \mathrm{ppm}(\mathrm{s}, 2 \mathrm{H}, \mathrm{CH} 2)$, at $5.2 \mathrm{ppm}(\mathrm{br}, 2 \mathrm{H}, \mathrm{NH} 2), 2 \mathrm{~b}$ at $9.0 \mathrm{ppm}(\mathrm{s}$, $1 \mathrm{H}, \mathrm{OH})$, at $8.7 \mathrm{ppm}(\mathrm{s}, 1 \mathrm{H}, \mathrm{SH})$, at $8.5 \mathrm{ppm}(\mathrm{s}, 1 \mathrm{H}, \mathrm{Hpyrimidine})$, at $6 \mathrm{ppm}(\mathrm{s}, 1 \mathrm{H}, \mathrm{CH})$, at $5.2 \mathrm{ppm}(\mathrm{br}, 2 \mathrm{H}, \mathrm{NH} 2)$, at $2.3 \mathrm{ppm}(\mathrm{s}, 3 \mathrm{H}, \mathrm{CH} 3), 2 \mathrm{c}$ : at $9.0 \mathrm{ppm}(\mathrm{s}, 1 \mathrm{H}, \mathrm{OH})$, at $8.7 \mathrm{ppm}(\mathrm{s}, 1 \mathrm{H}, \mathrm{SH})$, at $8.5 \mathrm{ppm}(\mathrm{s}, 1 \mathrm{H}, \mathrm{Hpyrimidine}), 6$ $\operatorname{ppm}(\mathrm{s}, 2 \mathrm{H}, \mathrm{CH} 2)$, at $5.2 \mathrm{ppm}(\mathrm{br}, 2 \mathrm{H}, \mathrm{NH} 2)$, at $8.3-7(\mathrm{~m}, 10 \mathrm{H}, \mathrm{Ar}-\mathrm{H}+)$. Iminophosphorane 2a-f reacted with aromatic isocyanates to give carbodiimides $3 \mathrm{a}-\mathrm{f}$ (Scheme 1). The structure of compounds $3 \mathrm{a}-\mathrm{f}$ were confirmed by elemental analysis (c.f. Table 1), and IR spectrum $(\mathrm{KBr})$, general absorption band at $3500 \mathrm{~cm}^{-1}(\mathrm{OH})$. The $1 \mathrm{H}$ NMR spectrum (DMSO)[10] of compounds 3a-f showed signals, 3a: at $9.0 \mathrm{ppm}(\mathrm{s}, 1 \mathrm{H}, \mathrm{OH})$, at $8.7 \mathrm{ppm}(\mathrm{s}, 1 \mathrm{H}$, $\mathrm{SH})$, at $8.5 \mathrm{ppm}(\mathrm{s}, 1 \mathrm{H}, \mathrm{Hpyrimidine}), 6 \mathrm{ppm}(\mathrm{s}, 2 \mathrm{H}, \mathrm{CH} 2)$, at $5.2 \mathrm{ppm}(\mathrm{br}, 2 \mathrm{H}, \mathrm{NH} 2), 3 \mathrm{~b}$ : at $9.0 \mathrm{ppm}(\mathrm{s}, 1 \mathrm{H}, \mathrm{OH})$, at at $8.7 \mathrm{ppm}(\mathrm{s}, 1 \mathrm{H}, \mathrm{SH})$, at $8.5 \mathrm{ppm}(\mathrm{s}, 1 \mathrm{H}, \mathrm{Hpyrimidine})$, at $6 \mathrm{ppm}(\mathrm{s}, 1 \mathrm{H}, \mathrm{CH})$, at $5.2 \mathrm{ppm}(\mathrm{br}, 2 \mathrm{H}, \mathrm{NH} 2)$, at 2.3 $\operatorname{ppm}(\mathrm{s}, 3 \mathrm{H}, \mathrm{CH} 3), 3 \mathrm{c}$ : at $9.0 \mathrm{ppm}(\mathrm{s}, 1 \mathrm{H}, \mathrm{OH})$, at $8.7 \mathrm{ppm}(\mathrm{s}, 1 \mathrm{H}, \mathrm{SH})$, at $8.3-7(\mathrm{~m}, 10 \mathrm{H}, \mathrm{Ar}-\mathrm{H}+)$, at $8.5 \mathrm{ppm}(\mathrm{s}, 1 \mathrm{H}$, Hpyrimidine), $6 \mathrm{ppm}(\mathrm{s}, 2 \mathrm{H}, \mathrm{CH} 2)$, at $5.2 \mathrm{ppm}(\mathrm{br}, 2 \mathrm{H}, \mathrm{NH} 2)$. The carbodiimides $3 \mathrm{a}-\mathrm{f}$ reacted with hydrazine to give the guanidine derivatives intermediate 4 via nucleophilic addition, which cyclizes to give compounds $5 \mathrm{a}-\mathrm{f}$ (Scheme 1). The structure of compounds 5a-f were confirmed by elemental analysis (c.f. Table 1), and IR spectrum $(\mathrm{KBr})$, general absorption band at $3500 \mathrm{~cm}^{-1}(\mathrm{OH})$, and $3370-3350 \mathrm{~cm}^{-1}(\mathrm{NH} 2, \mathrm{NH})$. The $1 \mathrm{H}$ NMR spectrum (DMSO)[10] of compounds $5 \mathrm{a}-\mathrm{f}$ showed signals, $5 \mathrm{a}$ : at $9.5(\mathrm{~s}, 1 \mathrm{H}, \mathrm{NH})$, at $9.0 \mathrm{ppm}(\mathrm{s}, 1 \mathrm{H}, \mathrm{OH})$, at 8.7 ppm(s, $1 \mathrm{H}, \mathrm{SH}) 8.5 \mathrm{ppm}(\mathrm{s}, 1 \mathrm{H}, \mathrm{Hpyrimidine})$, at $6 \mathrm{ppm}(\mathrm{s}, 2 \mathrm{H}, \mathrm{CH} 2)$, at $5.2 \mathrm{ppm}(\mathrm{br}, 2 \mathrm{H}, \mathrm{NH} 2), 5 \mathrm{~b}:$ at $9.0 \mathrm{ppm}(\mathrm{s}$, $1 \mathrm{H}, \mathrm{OH})$, at $8.7 \mathrm{ppm}(\mathrm{s}, 1 \mathrm{H}, \mathrm{SH})$, at $8.5 \mathrm{ppm}(\mathrm{s}, 1 \mathrm{H}, \mathrm{Hpyrimidine})$, at $6 \mathrm{ppm}(\mathrm{s}, 1 \mathrm{H}, \mathrm{CH})$, at $5.2 \mathrm{ppm}(\mathrm{br}, 2 \mathrm{H}, \mathrm{NH} 2)$, at $2.3 \mathrm{ppm}(\mathrm{s}, 3 \mathrm{H}, \mathrm{CH} 3), 5 \mathrm{c}$ : at $9.0 \mathrm{ppm}(\mathrm{s}, 1 \mathrm{H}, \mathrm{OH}), 8.7 \mathrm{ppm}(\mathrm{s}, 1 \mathrm{H}, \mathrm{SH})$, at $8.3-7 \mathrm{ppm}(\mathrm{m}, 10 \mathrm{H}, \mathrm{Ar}-\mathrm{H}+)$, at 8.5 $\mathrm{ppm}(\mathrm{s}, 1 \mathrm{H}, \mathrm{Hpyrimidine})$, at $6 \mathrm{ppm}(\mathrm{s}, 2 \mathrm{H}, \mathrm{CH} 2)$, at $5.8 \mathrm{ppm}(\mathrm{br}, 2 \mathrm{H}, \mathrm{NH} 2)$.

In conclusion, we have developed an efficient synthesis pyrimidinopyrimidine thione derivatives via aza-Wittig reactions. This method utilizes easily accessible starting materials and allows mild reaction conditions, straightforward product isolation and good yields.

\section{References}

Cushman, M., Sambaiah, T., Jin, G.., Illarionov, B., Fischer, M., \& Bacher, A. (2004). Design, synthesis, and evaluation of 9-D-ribitylamino-1,3,7,9-tetrahydro-2,6,8-purinetriones bearing alkyl phosphate and alpha,alpha-difluorophosphonate substituents as inhibitors of tiboflavin synthase and lumazine synthase. $J$. Org. Chem., 69, 601.

Daboun, H. A., \& El-Reedv, A. M. Z. (1983). Symposium on Malononitrile and Other Reactive Malono-Synthons, Cairo, 7 April 1983, 85. Naturforsch, 38b, 1686.

Depecker, G., Patino, N., Giorgio, C. D., Terreux, R., Cabrol-Bass, D., Bailly, C., ... Condom, R. (2004). Cyclic PNA-based compound directed against HIV-1 TAR RNA: Modelling, liquid-phase synthesis and TAR binding Org. Biomol. Chem., 2, 74. http://dx.doi.org/10.1039/B311775H

Haraguchi, K., Kubota, Y., \& Tanaka, H. (2004). Ring Opening of 1',2'-Epoxynucleosides with Aluminum Reagents: Stereoselective Entry to Ribonucleosides Substituted at the Anomeric Position. J. Org. Chem., 69, 1831.

He, P., Li, Z. F., Hou, Q. F., Wang, Y. L., \& Zhao, K. (2013). An efficient synthesis of 2,3,6,7tetrasubstituted 4,6-dihydro-4-oxo-3H-pyrrolo[3,4-d]pyrimidin-7-carbonitrilederivatives Arkivoc (iii), 199-209.

He, P., Wu, J., Hu, Y. G., Li, Z. F., Hou, Q. F., Wang, Y. L., ... Zhang, E. (2014). Efficient and Selective Construction of Pyrrolo[3,2-d]pyrimidine Derivatives. Bull. Korean Chem. Soc., 35(2), 1.

Kaska, W. C. (1983). Phosphorus ylides are not only important synthons of the Wittig reaction but have also been used extensively as alkyl-ligands Coord. Chem Rev, 48, 1 .

Lertpibulpanya, D., Marsden, S. P., Rodriguez-Garcia, I., \& Kilner, C. A. (2006). Asymmetric Aza-Wittig Reactions: Enantioselective Synthesis of $\beta$-Quaternary Azacycles Angew. Chem. Int. Ed., 45, 5000. http://dx.doi.org/10.1002/ange.200601383

López, J. L., Tárraga, A., \& Molina, P. (2007). Substitution and ring-opening reactions of an azasubstituted [5] ferrocenophane: preparation of 1,1'-unsymmetrically disubstituted ferrocenes. Arkivoc, iv, 39.

Marsden, S. P., Mc Gonagle, A. E., \& Mckeever-Abbas, B. (2008). Catalytic aza-Wittig Cyclizations for Heteroaromatic Synthesis Org. Lett., 10, 2589. http://dx.doi.org/10.1021/o1800921n

Molina, P., \& Vilaplana, M. J. (1994). Iminophosphoranes: Useful Building Blocks for the Preparation of 
Nitrogen-Containing Heterocycles Synthesis, 1197. http://dx.doi.org/10.1055/s-1994-25672

Palacies, F., Herran, E., Alonso, C., Rubiales, G., Lecea, B., Ayerbe, M., \& Cossio, F. P. (2006). Reaction of N -Vinylic Phosphazenes with $\alpha, \beta$-Unsaturated Aldehydes. Azatriene-Mediated Synthesis of Dihydropyridines and Pyridines Derived from $\beta$-Amino Acids. J. Org. CHem., $71,6020$. http://dx.doi.org/10.1021\%2fjo060775b

Palacios, F., Alonso, C., Aparicio, D., Rubiales, G., \& Santos, J. M. (2007). The aza-Wittig reaction: An efficient tool for the construction of carbon-nitrogen double bonds Tetrahedron, 63, 523.

Palacios, F., Herran, E., Allonse, C., \& Rubiales, G. (2007). Regioselective synthesis of dihydropyridines and pyridines derived from $\beta$-aminoacids from $\mathrm{N}$-vinylic phosphazenes Arkivoc, (iv), 397.

Ramazani, A., Kazemizadeh, A. R., Ahmadi, E., Noshiranzadeh, N., \& Souldozi, A. (2008). Synthesis and Reactions of Stabilized Phosphorus Ylides Curr. Org. Chem., 12, 59. http://dx.doi.org/10.1039/9781849730839-00001.

Ramazani, A., Kazemizadeh, A. R., Namadi, E., Slepokura, K., \& Lis, T. (2006). Synthesis and X-Ray Single Crystal Structure of Dialkyl 2-[1-(2,2-Dimethylpropionyl)-3,3-dimethyl-2-oxobutyl]-3(triphenylphosphoranylidene)succinates. Z. Naturforsch, 616, 1128.

Stolzenberg, H., Weinberger, B., Fehlhammer, W. P., Pühlhofer, F. G., \& Weiss, R. (2005). Free and Metal-Coordinated ( $N$-Isocyanimino)triphenylphosphorane: X-ray Structures and Selected Reactions. Eur. J. Inorg. Chem., 21, 4263. http://dx.doi.org/10.1002/ejic.200500196

Taylor, E. C., \& Patel, H. H. (1992). Synthesis of pyrazolo[3,4,-d]pyrimidine analogues of the potent antitumor agent $\quad \mathrm{N}-\{4-[2-(2-a m i n o-4(3 \mathrm{H})-$ oxo-7H-pyrrolo[2,3-d]pyrimidin-5-yl)ethyl]benzoyl $\}$-L-glutamic acid (LY231514). Tetrahedron, 48, 808.

Traxler, P., Bold, G., Frei, J., Lang, M., Lydon, N., Mett, H., ...Furet, P. (1997). Use of a Pharmacophore Model for the Design of EGF-R Tyrosine Kinase Inhibitors: 4-(Phenylamino)pyrazolo[3,4-d]pyrimidines. J. Med. Chem., 40, 3601. http://dx.doi.org/10.1021/jm970124v

Vicentini, C.B., Bli, T., Veronese, A.C., Brandolini, V., Manfrini, M., \& Guarneri, M. (1989). Synthesis and in-vitro antifungal activity of 6-trifluoromethyipyrazolo[3,4-6d]pyrimidin-4(5H)-thiones. Pestic, Sci., 27, 77. http://dx.doi.org/10.1002/ps.2780270108

Wnuk, S. F., Lewandowska, E., Companioni, D. R., Garcia, P. I., Jr., \& Secrist, J. A. (2004). Synthesis and cytotoxicity of 9-(2-deoxy-2-alkyldithio- $\beta$-D-arabinofuranosyl)purine nucleosides which are stable precursors to potential mechanistic probes of ribonucleotide reductases. III Org. Biomol. Chem., 2, 120. http://dx.doi.org/10.1039/B311504F

Wright, G. E., \& Brown, N. C. (1977). Inhibitors of Bacillus subtilis DNA polymerase III. Structure-activity relations of 6-(phenylhydrazino)uracils. J. Med. Chem., 20, 1181. http://dx.doi.org/10.1021/jm00219a014

\section{Copyrights}

Copyright for this article is retained by the author(s), with first publication rights granted to the journal.

This is an open-access article distributed under the terms and conditions of the Creative Commons Attribution license (http://creativecommons.org/licenses/by/3.0/). 